\title{
Anxiety in neurosurgical patients undergoing nonurgent surgery during the COVID-19 pandemic
}

\author{
*Francesco Doglietto, MD, PhD, ${ }^{1}$ Marika Vezzoli, PhD, ${ }^{2}$ Antonio Biroli, MD, ${ }^{1}$ \\ Giorgio Saraceno, MD, ${ }^{1}$ Luca Zanin, MD, ${ }^{1}$ Marta Pertichetti, ${ }^{3}$ Stefano Calza, PhD, ${ }^{2}$ \\ Edoardo Agosti, MD, ${ }^{1,4}$ Jahard Mijail Aliaga Arias, MD, ${ }^{1,5}$ Roberto Assietti, MD, ${ }^{6}$ \\ Silvio Bellocchi, MD, ${ }^{7}$ Claudio Bernucci, MD, ${ }^{8}$ Simona Bistazzoni, MD, PhD, ${ }^{7}$ \\ Daniele Bongetta, MD, PhD, ${ }^{6}$ Andrea Fanti, MD, ${ }^{8}$ Antonio Fioravanti, MD, ${ }^{5}$ \\ Alessandro Fiorindi, MD, PhD, ${ }^{1}$ Alberto Franzin, MD, ${ }^{9}$ Davide Locatelli, MD, ${ }^{4}$ \\ Raffaelino Pugliese, MD, ${ }^{10}$ Elena Roca, MD, ${ }^{1,9}$ Giovanni Marco Sicuri, MD, ${ }^{11}$ Roberto Stefini, MD, ${ }^{11}$ \\ Martina Venturini, MD, ${ }^{7}$ Oscar Vivaldi, MD, ${ }^{9}$ Costanza Zattra, MD, ${ }^{1,9}$ \\ Cesare Zoia, MD, PhD, ${ }^{10}$ and Marco Maria Fontanella, MD1
}

\begin{abstract}
${ }^{1}$ Neurosurgery, Department of Medical and Surgical Specialties, Radiological Sciences and Public Health, University of Brescia; ${ }^{2}$ Biostatistics, Department of Molecular and Translational Medicine, University of Brescia; ${ }^{2}$ Psychology, Domus Salutis, Brescia; ${ }^{4}$ Neurosurgery, University of Insubria, Varese; ${ }^{5}$ Neurosurgery, Cremona Hospital, Cremona; ${ }^{6}$ Fatebenefratelli Sacco Hospital, Milan; ${ }^{7}$ Neurosurgery, Sant'Anna Hospital, Como; ${ }^{8}$ Neurosurgery, Papa Giovanni XXIII Hospital, Bergamo; ${ }^{\circ}$ Neurosurgery,

Fondazione Poliambulanza Hospital, Brescia; ${ }^{10}$ Neurosurgery, Fondazione IRCCS Policlinico San Matteo, Pavia; and

${ }^{11}$ Neurosurgery, Legnano Hospital, Legnano, Italy
\end{abstract}

OBJECTIVE The COVID-19 pandemic has forced many countries into lockdown and has led to the postponement of nonurgent neurosurgical procedures. Although stress has been investigated during this pandemic, there are no reports on anxiety in neurosurgical patients undergoing nonurgent surgical procedures.

METHODS Neurosurgical patients admitted to hospitals in eastern Lombardy for nonurgent surgery after the lockdown prospectively completed a pre- and postoperative structured questionnaire. Recorded data included demographics, pathology, time on surgical waiting list, anxiety related to COVID-19, primary pathology and surgery, safety perception during hospital admission before and after surgery, and surgical outcomes. Anxiety was measured with the State-Trait Anxiety Inventory. Descriptive statistics were computed on the different variables and data were stratified according to pathology (oncological vs nononcological). Three different models were used to investigate which variables had the greatest impact on anxiety, oncological patients, and safety perception, respectively. Because the variables (Xs) were of a different nature (qualitative and quantitative), mostly asymmetrical, and related to outcome ( $Y$ ) by nonlinear relationships, a machine learning approach composed of three steps (1, random forest growing; 2 , relative variable importance measure; and 3, partial dependence plots) was chosen.

RESULTS One hundred twenty-three patients from 10 different hospitals were included in the study. None of the patients developed COVID-19 after surgery. State and trait anxiety were reported by $30.3 \%$ and $18.9 \%$ of patients, respectively. Higher values of state anxiety were documented in oncological compared to nononcological patients (46.7\% vs $25 \% ; p=0.055)$. Anxiety was strongly associated with worry about primary pathology, surgery, disease worsening, and with stress during waiting time, as expected. Worry about positivity to SARS-CoV-2, however, was the strongest factor associated with anxiety, even though none of the patients were infected. Neuro-oncological disease was associated with state anxiety and with worry about surgery and COVID-19. Increased bed distance and availability of hand sanitizer were associated with a feeling of safety.

ABBREVIATIONS MDA = mean decrease in accuracy; PDP = partial dependence plot; $\mathrm{RF}=$ random forest; STAI = State-Trait Anxiety Inventory; VIM = variable importance measure; $Y_{\mathrm{RF} 1}, Y_{\mathrm{RF} 2}, Y_{\mathrm{RF} 3}=$ the 3 outcomes used in random forests.

SUBMITTED August 1, 2020. ACCEPTED September 28, 2020.

INCLUDE WHEN CITING DOI: 10.3171/2020.9.FOCUS20681.

${ }^{*}$ F. Doglietto and M. Vezzoli contributed equally to this study. 
CONCLUSIONS These data underline the importance of psychological support, especially for neuro-oncological patients, during a pandemic.

https://thejns.org/doi/abs/10.3171/2020.9.FOCUS20681

KEYWORDS pandemic; anxiety; machine learning

$\mathrm{T}$ HE COVID-19 pandemic has forced many countries in the world into lockdown, ${ }^{1-3}$ including Italy, ${ }^{4-7}$ and has led to postponement of nonurgent surgical procedures to allow for reorganization of hospitals and ICUs. Furthermore, data from different research groups have demonstrated that COVID-19 is strongly associated with an increased risk of surgery-related mortality and complications, thus justifying a delay of surgery when the risk of infection is high. ${ }^{8-10}$

Italy is now in the post-lockdown phase of the COVID-19 pandemic. In Lombardy, which has been the most affected region, nonurgent neurosurgical procedures are now being scheduled, but surgery remains at a maximum $70 \%$ of prepandemic activity.

Although stress and anxiety during the COVID-19 pandemic is being investigated and dealt with in the general population, ${ }^{11}$ in neurosurgeons, ${ }^{12-14}$ and in some neurosurgical patients, ${ }^{15,16}$ to the best of our knowledge there are no reports on anxiety in neurosurgical patients who require nonurgent surgical procedures. The aim of this study was to investigate anxiety in neurosurgical patients undergoing nonurgent surgical procedures in the post-lockdown phase of the COVID-19 pandemic. Different data, including pathology, type of surgery, and trait and state anxiety, were collected in several hospitals, mainly located in eastern Lombardy, which is the area in Italy most affected by COVID-19. The primary objective of the paper was to quantify reported anxiety in neurosurgical patients; the secondary objectives were to investigate anxiety and its possible association with different factors, including fear of COVID-19 and underlying pathology.

\section{Methods}

Inclusion criteria for the study were as follows: adult patients undergoing nonurgent neurosurgical procedures who consented to study participation. Exclusion criteria were as follows: urgent/emergency surgery and age $<18$ years or unable to provide consent. The study was approved by the local ethics committee (study no. 4290; COVIDSAFENSG). Data were prospectively collected in a 1- to 4-week period in the different participating hospitals.

\section{Collected Data: Questionnaires and Clinical Data}

Each patient filled in 4 questionnaires: 3 before surgery and 1 after. Clinical data were provided by the neurosurgeon in charge of the patient in an additional data set.

The first questionnaire (see Supplemental Questionnaires) collected demographic data (age, sex, and highest academic degree); days surgical operation was postponed; and fear related to 1) disease, 2) COVID-19, and 3) hospitalization. Most answers are reported on a Likert scale from 1 (not at all) to 10 (very), avoiding the middle neutral option of odd scales.

The second and third questionnaires were the State-
Trait Anxiety Inventory (STAI), ${ }^{17-19}$ each based on 20 questions on a 4-point Likert scale. They measure two types of anxiety: 1) state anxiety (related to an event, e.g., a surgical procedure, in a specific moment in time; referred to henceforth as STAI-Y1); and 2) trait anxiety (the anxiety level usually perceived by the person; referred to henceforth as STAI-Y2). Each question is scored from a minimum of 1 to a maximum of 4 points. Hence, scores obtained from these 2 questionnaires (that measure the latent construct of anxiety) range from a minimum of 20 to a maximum of 80. Higher scores are positively correlated with higher levels of anxiety. In detail:

- $20 \leq$ STAI score < 48: Normal

- $48 \leq \mathrm{STAI} \leq 52$ : Mild Anxiety

- $52<\mathrm{STAI} \leq 80$ : Severe Anxiety

These questionnaires are widely used and have been validated in many languages, including Italian. ${ }^{20,21}$

The last questionnaire (see Supplemental Questionnaires), collected patients' impressions on safety from SARS-CoV-2 infection during hospitalization; perceived quality of the patient's experience in the neurosurgery ward and improvements obtained after surgery were also queried. As for the preoperative questionnaire, most answers were reported on a Likert scale from 1 to 10 .

The two novel questionnaires were tested at the beginning of June 2020 on an external and independent sample of 30 subjects in order to improve the questions' semantics and, consequently, their comprehension. Compilation time was less than 5 minutes per questionnaire and answers were collected with REDCap (a secure web application for building and managing online surveys and databases).

Clinical data included pathology, any prolongation of time on the waiting list, any postponement of hospital admission, duration and type of surgery, postoperative complications, surgical outcome, and days of hospitalization (Supplemental Table 6).

\section{Statistical Methods}

Descriptive statistics were computed on the different variables. In detail, the number of missing values (Nmiss); median; first quartile (Q1) and third quartile (Q3); range (minimum-maximum) for Likert scale; and additional mean and SD were calculated for quantitative variables; and the frequencies (absolute and percentage values) were computed for categorical variables.

Data were also stratified for pathology (oncological/ nononcological) to document any significantly different variables $(\mathrm{p}<0.05)$ in the two subpopulations. The Wilcoxon rank-sum test was used for Likert scales and quantitative variables; trend and chi-square tests were applied to ordered and qualitative variables, ${ }^{22}$ respectively. Moreover, the Pearson correlation coefficient (with 95\% CI and $\mathrm{p}$ value) was used to measure linear relationships between quantitative variables ${ }^{23}$ (in detail, between STAI-Y1 [state] and STAI-Y2 [trait] scores). 


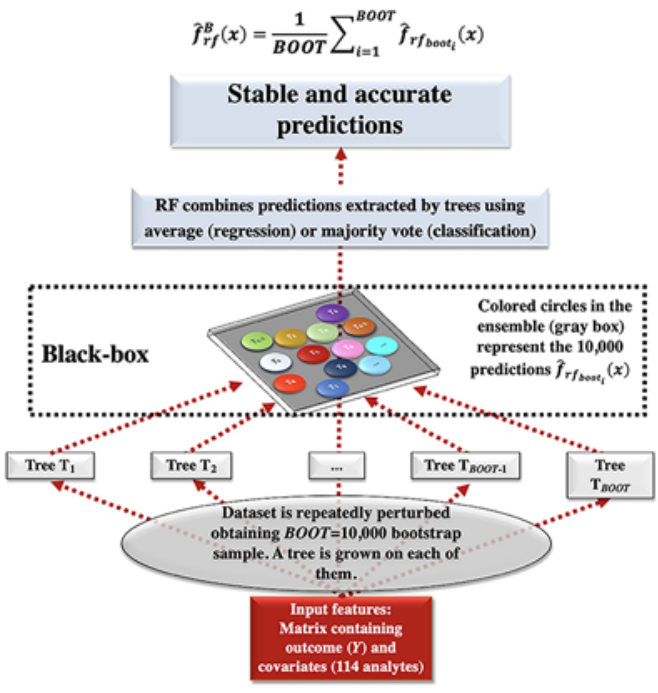

Step 1 - Random Forests

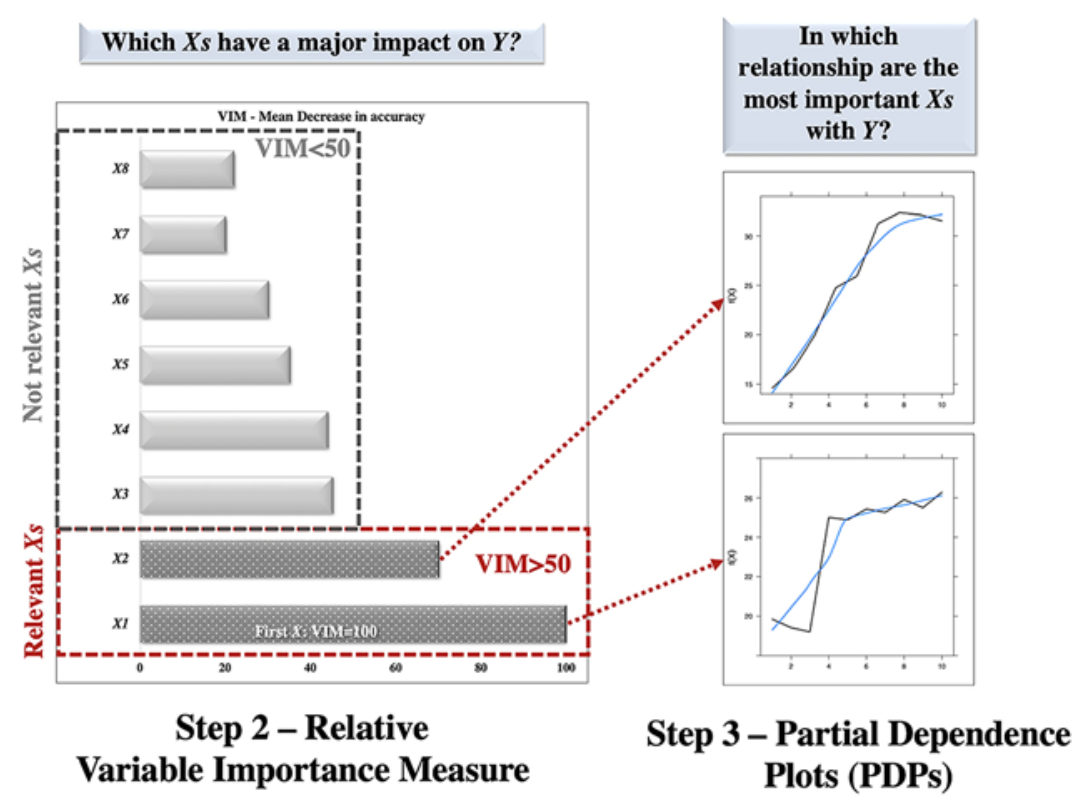

FIG. 1. The three-step procedure based on RF, VIM, and PDP. For each model that was estimated, a three-step procedure was followed. 1) An RF of 10,000 regression trees was grown. The figure should be read from the bottom to the top. The red box represents the data set. The algorithm perturbs the data set by means of bootstrap samples (procedure represented by a gray oval, where many samples of the same dimension of the original data set are repeatedly drawn, with replacement, from it) and, on each of them, grows a regression tree (gray rectangle) extracting single predictions (colored circles). These forecasts obtained by uncorrelated models are then combined with a simple mean, which produces predictions that are more accurate than the ones obtained by the single trees. 2) Using VIM, the MDA was extracted: this is a measure of variable importance that identifies which variables $(X s)$ are associated with the outcome $(Y)$ of the model. The MDA was also used to select the most significant variables (i.e., only those $X s$ with a VIM > 50). 3) For each selected variable the relationship between $X$ and $Y$ was visualized by a PDP. This $2 \mathrm{D}$ plot reports the values of the selected variable on the $\mathrm{x}$-axis and the values of the predictions on the $y$-axis. A smoothing function (linear, nonlinear, monotonic, nonmonotonic, etc.) is added to the graph (blue lines).

Three different models were used to identify which variables $(X)$ have the greatest impact on the outcomes $(Y)$. Because the variables were of a different nature (qualitative and quantitative), mostly asymmetrical, and related to $Y$ by nonlinear relationships, a machine learning approach was chosen: the random forest (RF), ${ }^{24}$ which is an ensemble method ${ }^{25-27}$ that combines thousands of regression trees. ${ }^{28}$ The idea behind the RF is quite simple: first, the algorithm perturbs the data set, generating many bootstrap samples and growing, on each of them, an overfitted decision tree (i.e., each terminal node contains few observations). The result of this procedure is a large number of uncorrelated models (this is due to the tree instability) that may not be accurate, but which combined together (with a simple mean) produce predictions that are more accurate than the ones obtained by single trees ${ }^{24}$ (Fig. 1-Step 1 shows, in a simple way, how the algorithm works; it should be read from the bottom to the top). This interesting effect is called "the wisdom of crowds"- each tree protects others from their individual errors.

Three RFs were grown:

1. RF1-state anxiety: state anxiety was modeled $\left[Y_{\mathrm{RF} 1}=\right.$ STAI-Y1 score $\left(20 \leq Y_{\mathrm{RF} 1} \leq 80\right)$ ] to investigate which concerns have a primary role for it. The matrix of covariates $(X)$ and corresponding questions of preoperative questionnaires are reported in Table 1.

2. RF2 - pathology: pathology was divided in a dicho- tomic variable (oncological/nononcological) and modeled $\left[Y_{\mathrm{RF} 2}=\right.$ pathology $]$. An RF of 10,000 regression trees was grown, because when $Y$ is dichotomic, regression instead of classification can be used ${ }^{29}$ The aim was to identify what concerns, collected by the preoperative questionnaire, most discriminate oncological from nononcological patients. The covariates and corresponding questions are reported in Table 2.

3. RF3 - protection: in this model the covariates that are associated with the protection from SARS-CoV-2 during hospital admission in neurosurgery were investigated. The covariates and corresponding questions from the postoperative questionnaire are reported in Table 3.

The RF algorithm was not used in predictive terms, but rather to highlight the relationships between covariates and outcomes. To clarify these relationships, two additional methods were used: 1) relative variable importance measure ${ }^{24}$ (VIM) and 2) partial dependence plots ${ }^{30}$ (PDPs).

The VIM identifies the covariates that most impact on the prediction of $Y$ and helps to make variable selection. In particular, the VIM called mean decrease in accuracy 24,31 (MDA) was used. The algorithm assigns a score of 100 to the variable most associated with the outcome, ordering the remaining variables from the most to the least important. The VIM, however, does not provide information on how the model predictions change according to $X$ values.

Therefore, the covariates with a VIM $>50$ were select- 
TABLE 1. Definitions of covariates $\left(X_{\mathrm{RF} 1}\right)$ used in RF1 where the outcome $\left(Y_{\mathrm{RF} 1}\right)$ is the STAI-Y1 score

\begin{tabular}{|c|c|c|}
\hline$X_{\mathrm{RF} 1}$ No. & Covariate Definition & Question No. in the Preop Questionnaire \\
\hline 1 & Worried about positivity for coronavirus. & 19 \\
\hline 2 & How worried are you about the pathology for which you have been admitted? & 20 \\
\hline 3 & How much are you worried about the surgical procedure? & 21 \\
\hline 4 & How anxious were you about a possible worsening of your condition? & 12 \\
\hline 5 & How stressed were you during the waiting time to admission? & 11 \\
\hline 6 & Age. & NA \\
\hline 7 & Becoming positive for SARS-CoV-2 during hospitalization. & 22 \\
\hline 8 & How many days would you have been willing to postpone your admission? & 13 \\
\hline 9 & How safe do you feel in the neurosurgical ward? & 17 \\
\hline 10 & Perception of time from neurosurgical evaluation to admission. & $14^{*}$ \\
\hline 11 & How much did COVID-19 increase concern about neurosurgery admission? & 10 \\
\hline 12 & How useful is the screening for SARS-CoV-2 performed preoperatively? & 15 \\
\hline 13 & How safe is the screening for SARS-CoV-2 performed preoperatively? & 16 \\
\hline
\end{tabular}

$\mathrm{NA}=$ not applicable.

The table reports the 13 covariates that were investigated in RF1. Covariate definition is reported with the corresponding question number from the preoperative questionnaire. The question is reported in full when it is significantly different from the covariate definition.

* The time span from your neurosurgical evaluation to the present admission felt: very long; long; short; very short.

ed and by means of PDPs (a 2D plot), the functional relationship between the selected covariates and predictions obtained from the model could be visualized. ${ }^{32}$ The PDP graph reports on the $\mathrm{x}$-axis the covariate under inspection and on the $\mathrm{y}$-axis the predictions. A smoothing function was added to the graph, because it is frequently performed.

Figure 1 provides a visual summary of this three-step, machine learning procedure based on RF, VIM, and PDP. All the analyses were performed with R 4.0.1 software.

\section{Results}

After exclusion of 11 patients due to significant missing data, 123 subjects $(\mathrm{M} / \mathrm{F}, 64 / 59$; mean age 60.28 years [SD 15.08 years]) were included in the study, with 114 variables for each (Supplemental Tables 3-6). Data on treating hospitals, highest education qualification, and previous admissions to neurosurgery are reported in Supplemental Tables 1-2.

Hospital admission to neurosurgery was postponed for a mean of 49.72 days (median 30, range $0-150$ days). The median values related to stress during the waiting time and anxiety about worsening were $7 / 10$ and $6 / 10$, respectively. The waiting time was reported as very long or long by $61 \%$ of patients, increasing to $73 \%$ when patients whose admission was not postponed $(n=35)$ were excluded. There were no statistically significant differences in perception of these features between nononcological and oncological patients; the latter had a significantly shorter waiting time for admission (median 121 vs 39 days; $\mathrm{p}=0.019$ ).

SARS-CoV-2 screening was considered useful and safe by the majority of patients (median 9/10 and 8/10, respectively). Oncological patients considered screening significantly less useful than nononcological patients (median $8 / 10$ vs $9 / 10$, respectively; $p=0.041$ ). All patients felt safe (median 9/10) in the neurosurgery ward.

The score extracted by STAI-Y1 and -Y2 questionnaires documented a median, which corresponds to "normal" (42 and 40 of 80 , respectively); mild anxiety was evident in 14 and 12 patients in STAI-Y1 and -Y2; severe anxiety was documented in 23 and 11 patients, respectively. STAI-Y1

TABLE 2. Definitions of covariates $\left(X_{R F 2}\right)$ used in RF2 where the outcome $\left(Y_{R F 2}\right)$ is the pathology

\begin{tabular}{clc}
\hline$X_{\mathrm{RF} 2}$ No. & \multicolumn{1}{c}{ Covariate Definition } & Question No. in the Preop Questionnaire \\
\hline 1 & STAI-Y1 score. & NA \\
\hline 2 & How much are you worried about the surgical procedure? & 21 \\
\hline 3 & What is the risk that you have been infected by SARS-CoV-2? & 18 \\
\hline 4 & How anxious were you about a possible worsening of your condition? & 12 \\
\hline 5 & Becoming positive for SARS-CoV-2 during hospitalization. & 22 \\
\hline 6 & How much has COVID-19 increased concern about neurosurgery admission? & 10 \\
\hline 7 & How worried are you about the pathology for which you have been admitted? & 20 \\
\hline 8 & Worried about positivity to coronavirus. & 19 \\
\hline
\end{tabular}

The table reports the 8 covariates that were investigated in RF2. Covariate definition is reported with the corresponding question number from the preoperative questionnaire. 
TABLE 3. Definitions of covariates $\left(X_{\mathrm{RF} 3}\right)$ used in $\mathrm{RF} 3$ where the outcome $\left(Y_{\mathrm{RF} 3}\right)$ is the protection

\begin{tabular}{|c|c|c|}
\hline$X_{\mathrm{RF} 3}$ No. & Covariate Definition & Question No. in the Postop Questionnaire \\
\hline 1 & Feeling of safety due to distance between beds. & 8 \\
\hline 2 & Feeling of safety due to hand sanitizer gel available in hospital. & 6 \\
\hline 3 & Feeling of safety due to health personnel following security protocols. & 9 \\
\hline 4 & Feelings of safety due to the procedures to prevent infection from SARS-CoV-2. & 12 \\
\hline 5 & Feeling of safety due to measurement of body temperature at hospital entrance. & 4 \\
\hline 6 & Feeling of safety due to masks. & 5 \\
\hline 7 & Feeling of safety due to a reassuring behavior of health personnel. & 10 \\
\hline 8 & Feeling of safety due to sanitization of hospital environments. & 7 \\
\hline 9 & In the operating room, did you feel safe from coronavirus? & 2 \\
\hline 10 & Did the health personnel seem prepared for the postoperative period? & 11 \\
\hline
\end{tabular}

The table reports the 10 covariates that were investigated in RF3. Covariate definition is reported with the corresponding question number from the postoperative questionnaire.

and -Y2 were positively correlated (Pearson's correlation coefficient $\rho=0.56 ; 95 \%$ CI $0.43-0.67$; $p<0.01)$. This shows that patients with higher levels of STAI-Y1 (state anxiety) also have higher levels of STAI-Y2 (trait anxiety). Whereas STAI-Y1 and -Y2 scores were comparable between nononcological and oncological patients, subjects with severe state anxiety were more frequent in the latter group: the difference did not reach statistical significance (16.30\% vs $26.67 \%$; $\mathrm{p}=0.055)$.

Patients reported being worried mainly about their pathology and surgery (median 7/10 for both), rather than the possibility of being positive for SARS-CoV-2 (median $2 / 10$ ) or contracting the disease during hospitalization (median 3/10). Oncological patients were significantly more worried about their pathology $(\mathrm{p}=0.019)$ and surgery $(\mathrm{p}=0.005)$.

The perceived risk of being infected by SARS-CoV-2 was quantified with a median of $15 / 100(\mathrm{Q} 1, \mathrm{Q} 3: 1.00$, 40.00) of the entire sample, with higher, although not statistically significant, values reported by oncological compared with nononcological patients (20 vs $10 ; \mathrm{p}=0.138$ ).

After surgery, most patients felt safe from SARSCoV-2 in the operating room $(97.50 \%)$ and during hospitalization (median 8/10). The different features that were investigated for a feeling of safety (e.g., hand sanitizer, masks, distance between beds) were all scored high, with medians between 8/10 and 9/10 (Supplemental Table 5). Cleaning and sanitization of the hospital environment was scored significantly lower by oncological patients when they were asked how much it contributed to the feeling of safety (median 8/10 vs 9/10; $\mathrm{p}=0.047$ ).

The experience in neurosurgery was rated as high by all patients (median 9/10), and most patients felt improved after surgery $(88.24 \%)$. Many were still worried about their pathology (median 7/10), and significantly more so if they had an oncological disease (median $8 / 10$ vs $7 / 10$; $\mathrm{p}=0.002$ ).

Surgical complications were relatively rare $(6.5 \%)$, but significantly more frequent in oncological patients (16.67\% vs $3.23 \%$; $p=0.009)$. Surgical duration, days in the ICU, and days in hospital were significantly longer in oncological patients (Supplemental Table 7).

\section{RF1-State Anxiety}

In the first RF, 10,000 regression trees were grown, obtaining stable and accurate predictions. Applying step 2 of the machine learning approach, the relative VIM reported in Fig. 2 (left panel) was obtained, reporting 6 covariates that are associated with state anxiety. The most important variable associated with state anxiety is the worry for positivity to coronavirus (relative VIM = 100). The relationship of the 6 covariates associated with state anxiety is visualized by means of PDPs in Fig. 2 (right panel). PDPs in Fig. 2A-E highlight nonlinear positive relationships: functions are nonmonotonic, but it is evident that higher values of the selected covariate correspond to higher values of state anxiety. The only variable that did not change according to state anxiety is age (PDP-Fig. 2F, right panel).

\section{RF2-Pathology}

In the second RF, the relative MDA VIM (left panel of Fig. 3) selected three variables as strongly associated with the pathology (i.e., oncological and nononcological), in order of importance: 1) state anxiety (VIM = 100); 2) worry about the surgical procedure (VIM $=60.20)$; and 3 ) risk of SARS-CoV-2 infection (VIM = 53.13). In all three cases, PDPs highlight nonlinear positive relationships: higher values of selected $X \mathrm{~s}$ (x-axis) correspond to a higher probability of being oncological patients (on the y-axis) (Fig. 3 , right panel).

\section{RF3-Protection}

In the third RF, the relative MDA VIM (left panel of Fig. 4) selected only two features as strongly associated with the feeling of protection, in order of importance: 1) distance between beds (VIM = 100); and 2) hand sanitizer gel (VIM =66.07). In both cases, PDPs highlight nonlinear positive relationships (right panel of Fig. 4): if the importance attributed to distance between beds and use of hand sanitizer gel (both on the X-axis) increases, patients feel more protected.

\section{Discussion}

To the best of our knowledge, this paper is the first to systematically address anxiety in neurosurgical patients 


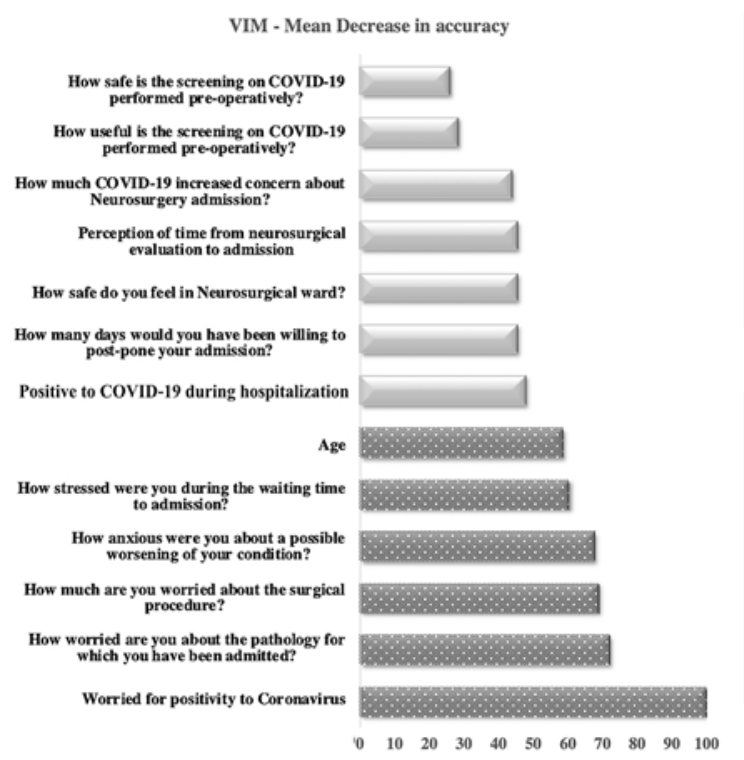

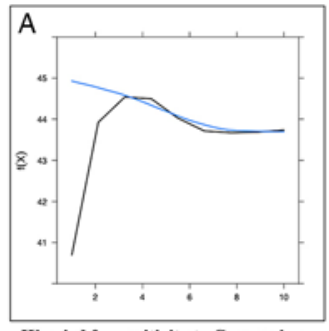

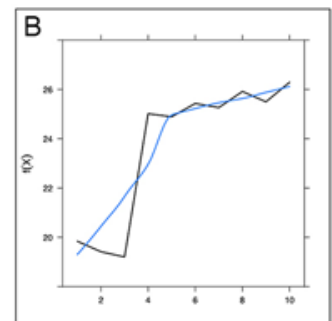

Worried for positivity to Coronavirus

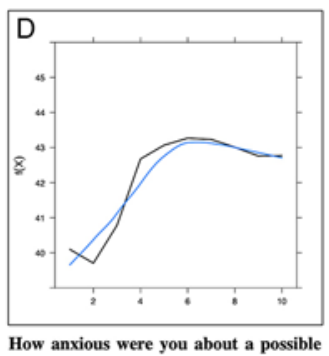

How anxious were you about
worsening of your condition?
How worried are you about the pathology for which you have been admitted?

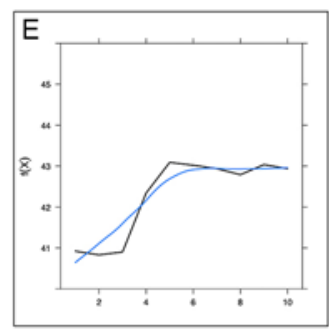

How stressed were you during the

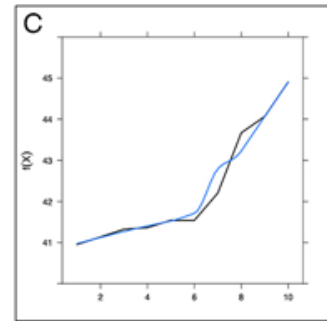

How much are you worried about the surgical procedure?

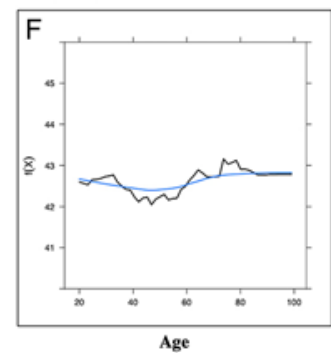

FIG. 2. Modeling of state anxiety (RF1). Relative VIM and PDPs of the first RF (RF1) are reported in the left and right panels, respectively. In the left panel, the 6 variables most associated with state anxiety (i.e., VIM $>50 \%$ ) are depicted by black bars with white dots, in order of importance: 1) worry about positivity to SARS-CoV-2; 2) worry about primary pathology; 3) worry about surgical procedure; 4) anxiety about clinical worsening; 5) stress of being on the waiting list; and 6) age. The other 7 variables that were less associated with state anxiety are reported in gray bars. In the right panel, the relationships between the 6 most associated variables and the predictions obtained by the RF1 are visualized by PDPs. Except for PDP (panel F, age), a nonlinear, positive relationship of each $X$ (on $X$-axis) with respect to the estimated STAI-Y1 score (on $y$-axis) is evident. A smoothing function is added to the graphs as a blue line.

VIM - Mean Decrease in accuracy

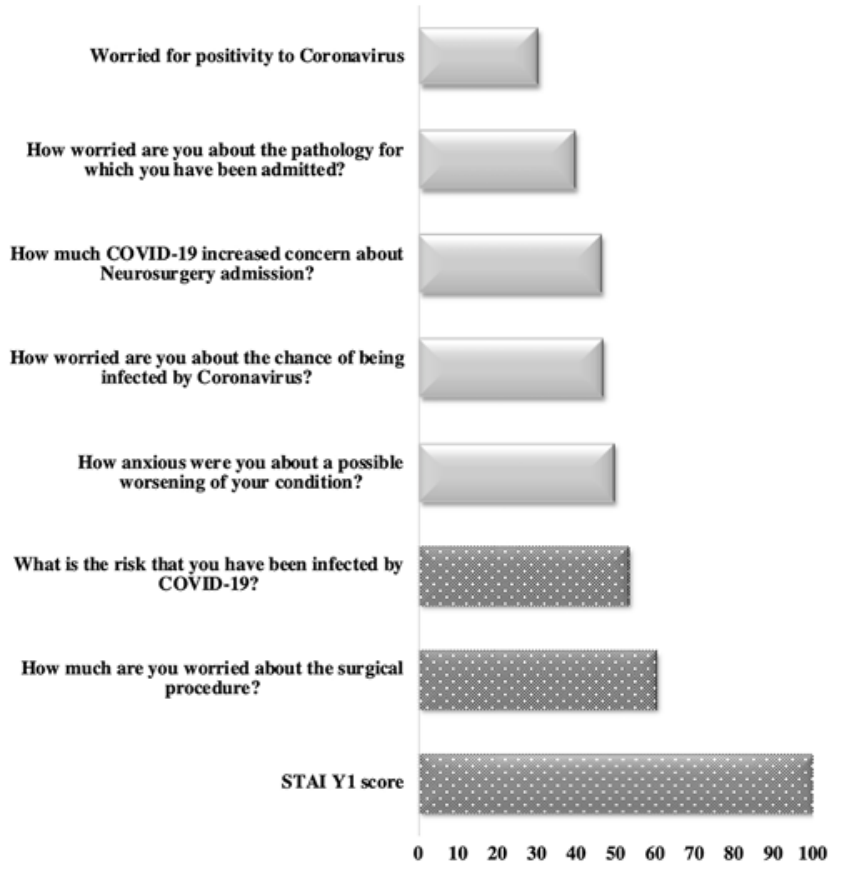

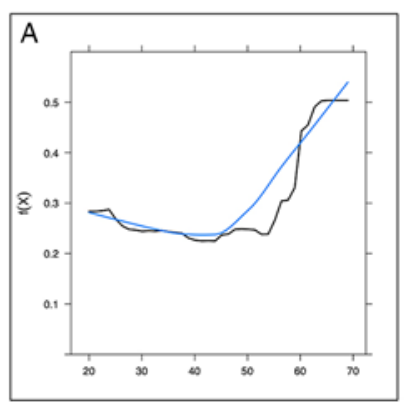

STAI Y1 score

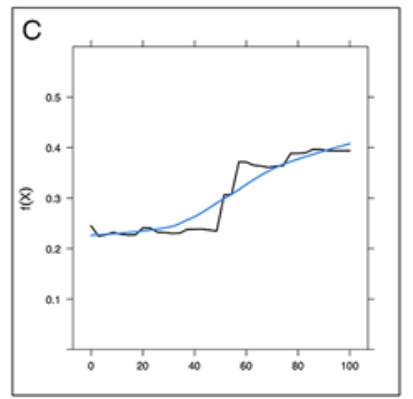

What is the risk that you have been infected by COVID-19?

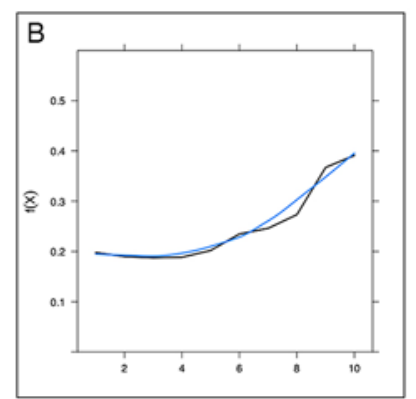

How much are you worried about the surgical procedure?

FIG. 3. Modeling of pathology (RF2). Relative VIM and PDPs of the second RF (RF2) are reported in left and right panels, respectively. The relative VIM extracted from RF2 shows the ranking from the most to least important variable and identifies the three covariates (black bars with white dots) that are strongly associated with pathology (oncological/nononcological patients). The gray bars describe the variables that are less associated (VIM $<50 \%$ ) with the pathology. The relationships between the three most associated variables and the predictions obtained by the RF2 are visualized by PDPs (right panel): in all cases a nonlinear, positive relationship is evident. The smoothing function is represented by the blue lines. 


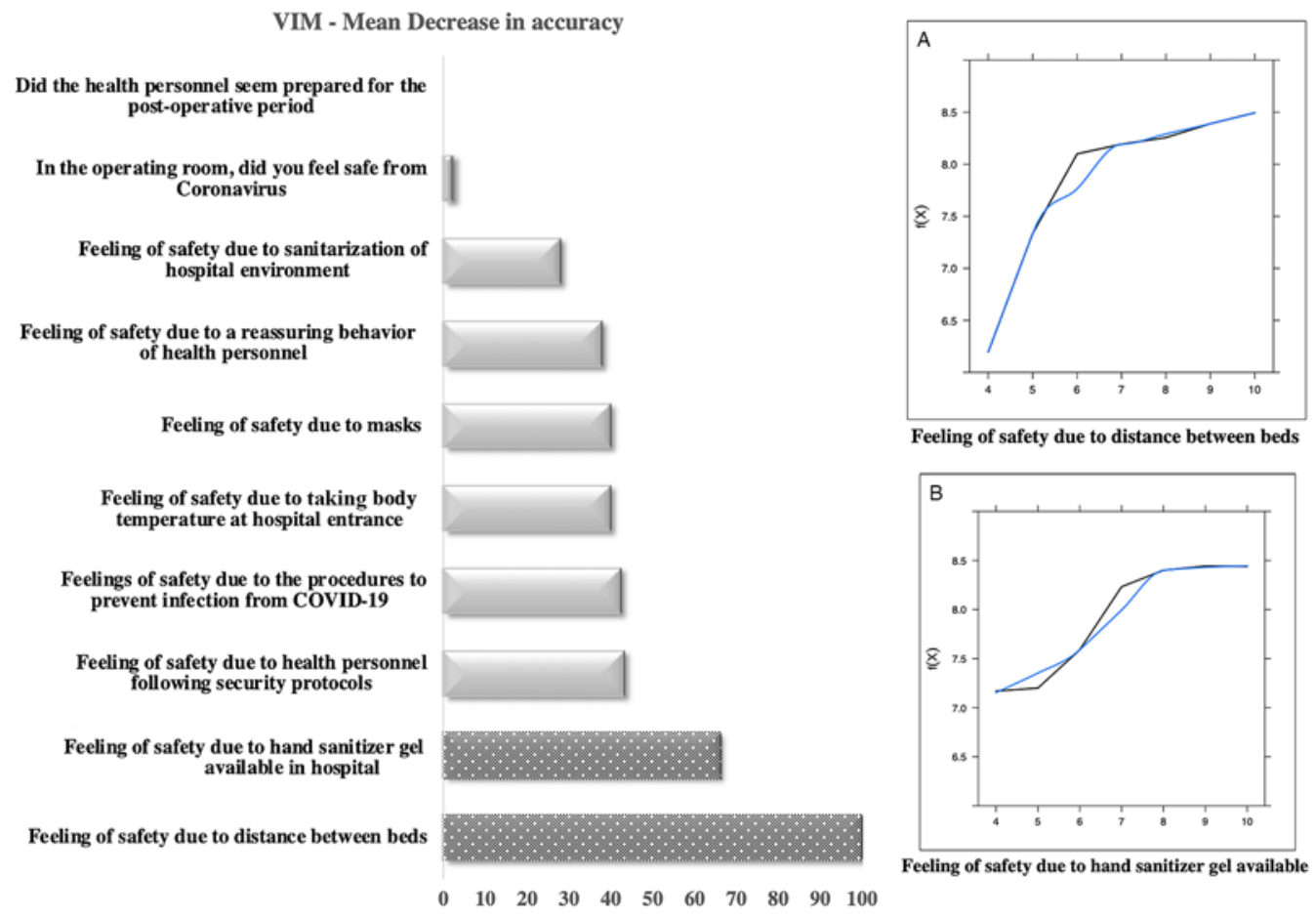

FIG. 4. Modeling of safety perception (RF3). Relative VIM and PDPs of the third RF (RF3) are reported in left and right panels, respectively. In the left panel, the relative MDA VIM extracted from RF3 shows that only 2 of 10 variables are most associated with the feeling of protection from SARS-CoV-2 during hospital admission in neurosurgery (black bars with white dots). Positive, nonlinear relationships are visualized by PDPs (right panel) between the two variables and the feeling of safety. The smoothing function is represented by blue lines.

who undergo surgical treatment during the COVID-19 pandemic. The study population comprised patients requiring nonurgent neurosurgical procedures who underwent treatment in 10 different hospitals located mainly in eastern Lombardy, an area that was severely involved in the COVID-19 outbreak in Italy. The study period is from 2-3 months after the end of the lockdown phase of the pandemic. All patients filled in a pre- and postoperative questionnaire, together with an evaluation of state and trait anxiety (i.e., STAI-Y1 and -Y2); type of pathology, surgery, complications, and outcomes were also prospectively recorded (see Supplemental Questionnaires).

Surgery was significantly postponed in this cohort of patients, mainly due to organizational issues (83\%) and only rarely $(2 / 123)$ for positivity to SARS-CoV-2. The waiting time was perceived as long or very long by the majority of patients (73\%). This is higher than previously reported, but the data are not really comparable because they are from other surgical specialties; ${ }^{33}$ no difference was documented between nononcological and oncological patients, even though the latter waited for a significantly shorter time. The stress due to the waiting time was reported as high (7/10): the fear of clinical worsening during the wait was scored as high as the worry for COVID-19 (6/10). The latter worry was indeed comparable to the one reported by the general population in the same study period. ${ }^{34}$

When state anxiety, measured by STAI-Y1, was considered, oncological patients were more severely stressed than nononcological patients (16\% vs $27 \%$ ). The differ- ence between the two groups for severe stress was somewhat different, but did not reach statistical difference (p $=0.055)$ : this might be due to the presence of patients affected by conditions such as vascular pathology $y^{35,36}$ or radiculopathy and disc herniation, ${ }^{37}$ which can both be associated with an increased state of anxiety. Modeling state anxiety (Fig. 2), it became evident that the patients' condition was significantly associated with the worry of being positive for SARS-CoV-2. This was the first variable identified by VIM, followed by intuitive ones such as the concern for the primary pathology, surgery, and worsening of their condition, as well as waiting time (Fig. 2). Anxiety and distress have been increasingly investigated and documented in neurosurgical patients in the last decade..$^{38-47}$

Fear of disease progression and related anxiety has been previously reported as frequent and of high clinical relevance for neurooncological patients. ${ }^{48-51}$ When pathology was dichotomized (oncological vs nononcological) and modeled, anxiety was identified by VIM as the first associated factor, followed by the worry about surgery, and then the perceived risk of being infected by SARS-CoV-2 . From a medical point of view, the increased stress for the pathology and surgery is justified by the recorded surgical time, ICU and hospital stay, as well as complications: all these were significantly higher in oncological compared to nononcological patients. The only factor that was not justified by medical data, because no patient was infected by SARS-CoV-2 in this series, was COVID-19-nonetheless, VIM identified it as the first factor associated with 
state anxiety. Interestingly, oncological patients considered screening for SARS-CoV-2 significantly less useful than nononcological patients; furthermore, cleaning and sanitization of the hospital environment was scored significantly lower in oncological compared to nononcological patients as a factor that transmitted a feeling of safety. These data might be explained by higher stress levels in oncological patients, leading to a more negative perception of the surrounding environment and, in this case, even of protective measures from the infectious disease causing the pandemic.

The association of anxiety with neuro-oncological disease and surgical intervention has been previously described. ${ }^{52-55}$ This study confirms that association and documents the association of severe stress in neuro-oncological patients with COVID-19. Anxiety and increased level of psychological distress have indeed been recently demonstrated in other oncological patients during the COVID-19 pandemic. ${ }^{56-59}$ Some authors have also underlined how COVID-19 might delay diagnosis and influence treatment and decision-making in oncology. ${ }^{60-62}$

We believe that the high prevalence of stress documented in this study, related not only to the neurosurgical disease but also to the pandemic, underlines the importance of dealing with it to improve patients' quality of life. Preoperative anxiety might be reduced by procedural information leaflets and behavioral training, ${ }^{63}$ but the pandemic should be recognized to best address the issue. Our data confirm that psychological support should be enhanced during outbreaks, ${ }^{62}$ possibly using novel solutions to provide follow-up care remotely during waiting times. . $^{58,60}$ Access to counseling services may mitigate some of the anxiety and depression experienced, ${ }^{56}$ and should be prioritized for those who might be experiencing distress even under normal circumstances. ${ }^{58}$

This study also investigated the feeling of safety conveyed by different features that were activated in all Italian hospitals during the pandemic. Interestingly, the increased distance between surgical beds was the first factor associated with a feeling of safety from SARS-CoV-2, followed by the availability of hand sanitizers. These data might be interpreted as a result of the ongoing social media communication on the importance of social distancing; we believe they might be important for hospital managers and to optimize communication with patients during this pandemic.

Finally, from a methodological point of view, it is important to note that the nonlinear and nonmonotonic relationships highlighted in PDPs (Figs. 2-4) justify the choice of RF methodology to describe the collected data. In fact, in this study we dealt with extremely asymmetrical variables of different natures (qualitative, ordinal and nominal, and quantitative): nonparametric methods are an effective and interesting alternative to extract information from such data.

\section{Limitations of the Study}

Anxiety was investigated only during the post-lockdown phase of the COVID-19 pandemic in eastern Lombardy, Italy; a control group before COVID-19 is not available. The recorded responses are probably influenced both by culture and the pandemic phase; they might change in different cultures and different phases of a pandemic. Nonetheless, even though the study was performed in the post-lockdown phase, and in a month that had experienced the fewest deaths due to COVID-19 since the first cases were recorded, anxiety in all neurosurgical patients was strongly associated with COVID-19; furthermore, anxiety was significantly higher in neuro-oncological patients. We believe this scenario might apply to different cultures, because reported anxiety before surgery is different but always relatively high in the general population in different countries. ${ }^{34}$ Unfortunately, psychological support was not systematically provided to our patients, which meant that this study could not quantify its potential benefit.

\section{Conclusions}

This study documented high levels of stress in neurosurgical patients undergoing nonurgent surgical procedures during the post-lockdown phase of the COVID-19 pandemic. Waiting time was perceived as long by most patients. Stress was associated with pathology, surgery, and fear of clinical worsening, but also, and most significantly, with fear of COVID-19. The distance between hospital beds and availability of hand sanitizers were associated with a feeling of safety. We believe that these data show the importance of psychological support that should always be part of neurosurgical practice, even more importantly during a pandemic.

\section{Acknowledgments}

We thank Luca Savona and Alessio Becchetti, who reviewed the first version of the questionnaires and provided insightful comments to improve their comprehension by the general Italian population.

\section{References}

1. Fontanella MM, De Maria L, Zanin L, et al. Neurosurgical practice during the severe acute respiratory syndrome coronavirus 2 (SARS-CoV-2) pandemic: a worldwide survey. World Neurosurg. 2020;139:e818-e826.

2. Fontanella MM, Saraceno G, Lei T, et al. Neurosurgical activity during COVID-19 pandemic: an expert opinion from China, South Korea, Italy, United Stated of America, Colombia and United Kingdom. J Neurosurg Sci. 2020;64(4):383-388.

3. Mathiesen T, Arraez M, Asser T, et al. A snapshot of European neurosurgery December 2019 vs. March 2020: just before and during the Covid-19 pandemic. Acta Neurochir (Wien). 2020;162(9):2221-2233.

4. Bernucci C, Brembilla C, Veiceschi P. Effects of the COVID-19 outbreak in Northern Italy: perspectives from the Bergamo Neurosurgery Department. World Neurosurg. 2020; 137:465-468.e1.

5. Cenzato M, DiMeco F, Fontanella M, et al. Editorial. Neurosurgery in the storm of COVID-19: suggestions from the Lombardy region, Italy (ex malo bonum). J Neurosurg. 2020; 133(1):33-34.

6. Perin A, Servadei F, DiMeco F; 'Hub and Spoke' Lombardy Neurosurgery Group. May we deliver neuro-oncology in difficult times (e.g. COVID-19)? J Neurooncol. 2020;148(1): 203-205.

7. Zoia $\mathrm{C}$, Bongetta $\mathrm{D}$, Veiceschi $\mathrm{P}$, et al. Neurosurgery during the COVID-19 pandemic: update from Lombardy, northern Italy. Acta Neurochir (Wien). 2020;162(6):1221-1222.

8. Aminian A, Safari S, Razeghian-Jahromi A, et al. COVID-19 
outbreak and surgical practice: unexpected fatality in perioperative period. Ann Surg. 2020;272(1):e27-e29.

9. Doglietto F, Vezzoli M, Gheza F, et al. Factors associated with surgical mortality and complications among patients with and without coronavirus disease 2019 (COVID-19) in Italy. JAMA Surg. 2020;155(8):691-702.

10. Nepogodiev D, Bhangu A, Glasbey JC, et al. Mortality and pulmonary complications in patients undergoing surgery with perioperative SARS-CoV-2 infection: an international cohort study. Lancet. 2020;396(10243):27-38.

11. World Health Organization. Mental health and COVID-19. Accessed October 23, 2020. https://www.who.int/teams/ mental-health-and-substance-use/covid-19

12. Benzel E. Emotional health in the midst of the coronavirus disease 2019 (COVID-19) pandemic. World Neurosurg. 2020; 138:xxv-xxvi.

13. Ganau M, Netuka D, Broekman M, et al. Neurosurgeons and the fight with COVID-19: a position statement from the EANS Individual Membership Committee. Acta Neurochir (Wien). 2020;162(8):1777-1782.

14. Sharif S, Amin F, Hafiz M, et al. COVID 19-depression and neurosurgeons. World Neurosurg. 2020;140:e401-e410.

15. Prasad S, Holla VV, Neeraja K, et al. Parkinson's disease and COVID-19: perceptions and implications in patients and caregivers. Mov Disord. 2020;35(6):912-914.

16. Salari M, Zali A, Ashrafi F, et al. Incidence of anxiety in Parkinson's disease during the coronavirus disease (COVID-19) pandemic. Mov Disord. 2020;35(7):1095-1096.

17. Spielberger CD. State-Trait Anxiety Inventory. In: Weiner IB, Craighead WE, eds. The Corsini Encyclopedia of Psychology. John Wiley \& Sons; 2010.

18. Spielberger CD, Gorsuch RL, Lushene RE, et al. Manual for the State-Trait Anxiety Inventory (Form Y1-Y2). Vol IV. Consulting Psychologists Press; 1983.

19. American Psychological Association. The State-Trait Anxiety Inventory (STAI). Accessed October 22, 2020. https: //www.apa.org/pi/about/publications/caregivers/practicesettings/assessment/tools/trait-state

20. Julian LJ. Measures of anxiety: State-Trait Anxiety Inventory (STAI), Beck Anxiety Inventory (BAI), and Hospital Anxiety and Depression Scale-Anxiety (HADS-A). Arthritis Care Res (Hoboken). 2011;63(suppl 11):S467-S472.

21. Pedrabissi L, Santinello M. Verifica della validità dello STAI forma Y di Spielberger. Giunti Organizzazioni Speciali. 1989;191-192:11-14.

22. Quiros-Roldan E, Castelli F, Lanza P, et al. The impact of antiretroviral therapy on iron homeostasis and inflammation markers in HIV-infected patients with mild anemia.J Transl Med. 2017;15(1):256.

23. Dancelli L, Manisera M, Vezzoli M. On two classes of weighted rank correlation measures deriving from the Spearman's $\rho$. In: Giudici P, Ingrassia S, Vichi M, eds. Statistical Models for Data Analysis. Studies in Classification, Data Analysis, and Knowledge Organization. Springer International Publishing; 2013:107-114.

24. Breiman L. Random forests. Mach Learn. 2001;45(1):5-32.

25. Manasse P, Savona R, Vezzoli M. Danger zones for banking crises in emerging markets. Int J Finance Econ. 2016;21(4): 360-381.

26. Vezzoli M. Exploring the facets of overall job satisfaction through a novel ensemble learning. Electron J Appl Stat Anal. 2011;4(1):23-38.

27. Vezzoli M, Ravaggi A, Zanotti L, et al. RERT: a novel regression tree approach to predict extrauterine disease in endometrial carcinoma patients. Sci Rep. 2017;7(1):10528.

28. Breiman L, Friedman J, Stone CJ, Olshen RA. Classification and Regression Trees. Taylor \& Francis; 1984.

29. Savona R, Vezzoli M. Fitting and forecasting sovereign defaults using multiple risk signals. Oxf Bull Econ Stat. 2015; 77(1):66-92.
30. Friedman JH. Greedy function approximation: a gradient boosting machine. Ann Stat. 2001;29(5):1189-1232.

31. Vezzoli M, Zuccolotto P. CRAGGING measures of variable importance for data with hierarchical structure. In: Ingrassia S, Rocci R, Vichi M, eds. New Perspectives in Statistical Modeling and Data Analysis. Studies in Classification, Data Analysis, and Knowledge Organization. Springer; 2011:393-400.

32. Carpita M, Vezzoli M. Statistical evidence of the subjective work quality: the fairness drivers of the job satisfaction. Electron J Appl Stat Anal. 2012;5(1):89-107.

33. Carr T, Teucher U, Mann J, Casson AG. Waiting for surgery from the patient perspective. Psychol Res Behav Manag. 2009;2:107-119.

34. COVID-19 fears. YouGov. Accessed October 22, 2020. https://yougov.co.uk/topics/international/articles-reports/2020/03/17/fear-catching-covid-19

35. Kubo Y, Ogasawara K, Kashimura H, et al. Cognitive function and anxiety before and after surgery for asymptomatic unruptured intracranial aneurysms in elderly patients. World Neurosurg. 2010;73(4):350-353.

36. Pala A, Pawlikowski A, Brand C, et al. Quality of life after treatment of unruptured intracranial aneurysms. World Neurosurg. 2019;121:e54-e59.

37. D'Angelo C, Mirijello A, Ferrulli A, et al. Role of trait anxiety in persistent radicular pain after surgery for lumbar disc herniation: a 1-year longitudinal study. Neurosurgery. 2010;67(2):265-271.

38. Bao Y, Li L, Guan Y, et al. Prevalence and associated positive psychological variables of anxiety and depression among patients with central nervous system tumors in China: a cross-sectional study. Psychooncology. 2017;26(2):262-269.

39. Bunevicius A, Deltuva V, Tamasauskas S, et al. Screening for psychological distress in neurosurgical brain tumor patients using the Patient Health Questionnaire-2. Psychooncology. 2013;22(8):1895-1900.

40. Gjerde PB, Litleskare S, Lura NG, et al. Anxiety and depression in patients with intracranial arachnoid cysts-a prospective study. World Neurosurg. 2019;132:e645-e653.

41. Goebel S, Stark AM, Kaup L, et al. Distress in patients with newly diagnosed brain tumours. Psychooncology. 2011;20(6): $623-630$

42. Goebel S, Mehdorn HM. Development of anxiety and depression in patients with benign intracranial meningiomas: a prospective long-term study. Support Care Cancer. 2013;21(5): 1365-1372.

43. Hoffmann K, Kamp M, Steiger H-J, et al. Correlation of psychooncological distress-screening and quality of life assessment in neurosurgical patients. Oncotarget. 2017;8(67): 111396-111404.

44. Moreale R, Campanella F, Marin F, et al. Emotional concerns and coping strategies in low grade glioma patients and reliability of their caregivers in reporting these concerns: findings from a cross-sectional study. Eur J Oncol Nurs. 2017; 30:113-119.

45. Rapp M, Schipmann S, Hoffmann K, et al. Impact of distress screening algorithm for psycho-oncological needs in neurosurgical patients. Oncotarget. 2018;9(60):31650-31663.

46. van der Vossen S, Schepers VPM, Berkelbach van der Sprenkel JW, et al. Cognitive and emotional problems in patients after cerebral meningioma surgery. J Rehabil Med. 2014; 46(5):430-437.

47. von Vogelsang A-C, Forsberg C, Svensson M, Wengström Y Patients experience high levels of anxiety 2 years following aneurysmal subarachnoid hemorrhage. World Neurosurg. 2015;83(6):1090-1097.

48. Eggen AC, Reyners AKL, Shen G, et al. Death anxiety in patients with metastatic non-small cell lung cancer with and without brain metastases. J Pain Symptom Manage. 2020; 60(2):422-429.e1.

49. Goebel S, Kaup L, Mehdorn HM. Measuring preoperative 
anxiety in patients with intracranial tumors: the Amsterdam preoperative anxiety and information scale. J Neurosurg Anesthesiol. 2011;23(4):297-303.

50. Goebel S, Mehdorn HM. Fear of disease progression in adult ambulatory patients with brain cancer: prevalence and clinical correlates. Support Care Cancer. 2019;27(9):3521-3529.

51. Loughan AR, Lanoye A, Aslanzadeh FJ, et al. Fear of cancer recurrence and death anxiety: unaddressed concerns for adult neuro-oncology patients. J Clin Psychol Med Settings. Published online December 17, 2019. doi:10.1007/s10880-01909690-8

52. Arnold SD, Forman LM, Brigidi BD, et al. Evaluation and characterization of generalized anxiety and depression in patients with primary brain tumors. Neuro Oncol. 2008;10(2): 171-181.

53. Dhandapani M, Gupta S, Mohanty M, et al. Prevalence and trends in the neuropsychological burden of patients having intracranial tumors with respect to neurosurgical intervention. Ann Neurosci. 2017;24(2):105-110.

54. Lucchiari C, Botturi A, Silvani A, et al. Cognitive strategies and quality of life of patients with high-grade glioma. Support Care Cancer. 2015;23(12):3427-3435.

55. Seibl-Leven M, von Reeken C, Goldbrunner R, et al. Clinical routine assessment of palliative care symptoms and concerns and caregiver burden in glioblastoma patients: an explorative field study. J Neurooncol. 2018;138(2):321-333.

56. Frey MK, Ellis AE, Zeligs K, et al. Impact of the COVID-19 pandemic on quality of life for women with ovarian cancer. Am J Obstet Gynecol. 2020;223(5):725.e1-725.e9.

57. Juanjuan L, Santa-Maria CA, Hongfang F, et al. Patientreported outcomes of patients with breast cancer during the COVID-19 outbreak in the epicenter of China: a cross-sectional survey study. Clin Breast Cancer. 2020;20(5):e651-e662.

58. Košir U, Loades M, Wild J, et al. The impact of COVID-19 on the cancer care of adolescents and young adults and their wellbeing: results from an online survey conducted in the early stages of the pandemic. Cancer. 2020;126(19):4414-4422.

59. Spence D. Isolated already, how COVID-19 has exacerbated anxiety for Australian cancer patients. Psychooncology. Published online July 16, 2020. doi:10.1002/pon.5478

60. Karacin C, Bilgetekin I. B Basal F, Oksuzoglu OB. How does COVID-19 fear and anxiety affect chemotherapy adherence in patients with cancer? Future Oncol. 2020;16(29):22832293.
61. Molica M, Mazzone C, Cordone I, et al. SARS-CoV-2 infection anxieties and general population restrictions delay diagnosis and treatment of acute haematological malignancies. $\mathrm{Br}$ J Haematol. 2020;190(1):e5-e8.

62. Vanni G, Materazzo M, Pellicciaro M, et al. Breast cancer and COVID-19: the effect of fear on patients' decision-making process. In Vivo. 2020;34(3)(suppl):1651-1659.

63. Tulloch I, Rubin JS. Assessment and management of preoperative anxiety. $J$ Voice. 2019;33(5):691-696.

\section{Disclosures}

The authors report no conflict of interest concerning the materials or methods used in this study or the findings specified in this paper.

\section{Author Contributions}

Conception and design: Fontanella, Doglietto, Pertichetti. Acquisition of data: Saraceno, Zanin, Agosti, Aliaga Arias, Assietti, Bistazzoni, Bongetta, Fanti, Roca, Sicuri, Stefini, Venturini, Zattra, Zoia. Analysis and interpretation of data: Doglietto, Vezzoli, Calza. Drafting the article: Doglietto. Critically revising the article: Fontanella, Doglietto, Vezzoli, Calza. Reviewed submitted version of manuscript: Fontanella, Biroli, Calza, Fiorindi. Approved the final version of the manuscript on behalf of all authors: Fontanella. Statistical analysis: Vezzoli, Calza. Administrative/technical/material support: Fontanella, Doglietto, Biroli, Calza, Bellocchi, Bernucci, Fioravanti, Franzin, Locatelli, Pugliese, Vivaldi, Zoia. Study supervision: Fontanella, Doglietto, Vezzoli, Biroli, Calza, Bellocchi, Bernucci, Fioravanti, Franzin, Locatelli, Vivaldi.

\section{Supplemental Information}

\section{Online-Only Content}

Supplemental material is available online. Supplemental Tables 1-7. https://thejns.org/doi/suppl/10.3171/ 2020.9.FOCUS20681.

Supplemental Questionnaires. https://thejns.org/doi/suppl/ 10.3171/2020.9.FOCUS20681.

\section{Correspondence}

Marco Maria Fontanella: University of Brescia, Italy. marco. fontanella@unibs.it. 